\title{
A novel method to chromatographically resolution of sulphonamides by vapour-programmed thin-layer chromatography
}

\begin{abstract}
In pharmaceutical sulphonamides, vapour-programmed thin-layer chromatography (VP-TLC) was implemented using Silica $\mathrm{Gel}_{\mathrm{GF}_{254}}$ in the separation and characterisation of sulphonamides. The outcomes of this method have been compared with thin layer chromatographic (TLC) process. 15 chemical substances were resolved in a neutral and alkali media. Results are far from optimal. With ether-methanol (90:10) the first seven substances are reasonably separated but the remaining eight all show about the same migration. Furthermore, it should be noticed that only about $3 / 5$ of the plate is utilised for the spread. Unsaturated chambers did not improve the separation significantly. Only sulphamethoxypyridazine and sulphisoxazole did not separate under these conditions, but the other components are well separated and migrated throughout the whole lane. It should be noted that the VP-systems described are suitable for two-dimensional development. Thus, it can be concluded that VP-TLC is highly effective in the analysis of sulphonamides.
\end{abstract}

Keywords: thin layer chromatographic, sulphamethoxypyridazine, pharmaceutical analysis, anaesthetics, amphoteric
Volume I Issue 4 - 2017

\author{
Ala Eldin Choukaife, Loai Aljerf \\ Department of Life Sciences, University of Damascus, Syria \\ Correspondence: Ala Eldin Choukaife, Department of \\ Life Sciences, Faculty of Dentistry, Syrian Private University, \\ Damascus, Syria, Tel +963-94 4490 I27, \\ Email chokala60@yahoo.com
}

Received: August 17, 2017| Published:September 07, 2017

\section{Introduction}

Identification, especially of drugs, plays an important role in pharmaceutical analysis. Thin-layer chromatography (TLC) has been widely applied in this field, but sometimes its resolving power leaves something to be desired. Barbiturates, steroids, sulphonamides and local anaesthetics, for example, represent classes where an abundance of closely similar derivatives are available. Separations and identification within these classes are by no means easy to perform. Recently, however, a new type of TLC was developed in our laboratory, namely vapour-programmed TLC (VP-TLC), ${ }^{1}$ which allows optimum vapour conditions to be established at the distinct feed tray and, as a consequence, better separations usually result. This technique has already proved to be very satisfactory for the analysis of barbiturates and local anaesthetics and preliminary investigations with a small number of sulphonamides were also very promising. ${ }^{2}$ We would now like to report a VP-TLC procedure by which fifteen sulphonamides can be separated and identified.

\section{Experimental}

\section{Solvents and standards}

All developing solvent components were reagent grade (Merck). Liquid compositions are given by volume. Solutions of sulphonamides $(0.1 \% \mathrm{w} / \mathrm{v})$ in acetone were used as standards. A coloured substance, 4-nitroaniline, was used as a reference compound.

\section{TLC apparatus and procedures}

Silica Gel $\mathrm{GF}_{254}$ (Merck) was used as adsorbent $-30 \mathrm{~g} / 60 \mathrm{ml}$ of demineralised water to prepare 5 plates, $20 \times 20 \mathrm{~cm}$, for a layer thickness of $0.25 \mathrm{~mm}$. After spreading (Desaga apparatus) the plates were air dried for $15 \mathrm{~min}$, heated for $30 \mathrm{~min}$ at $110^{\circ} \mathrm{C}$ in an oven with a fan, then cooled and stored in a desiccator. Samples, $3 \mu \mathrm{g}$, were applied to the plates and dried with the aid of a cold air blower, $2.5 \mathrm{~cm}$ from the lower edge of the plate and $1 \mathrm{~cm}$ apart. For VP-TLC strips of the gel, $0.5 \mathrm{~cm}$ wide at the sides and the lower edge, were removed from the plates. After spotting, the plates were left in the ambient atmosphere for at least $15 \mathrm{~min}$ to ensure water vapour adsorption in equilibrium with the ambient relative humidity. A VP-chamber (manufactured by Desaga) (Scheme 1) was used for development, while normal tank chambers (N-chambers, Shandon) were used as controls. N-chambers are cuboids twin-trough chambers. The trough chamber was made of rolled brass and the troughs were directly milled out. The top surface of the trough chamber was also milled to obtain a completely flat surface. After milling, the trough chamber and the other brass parts are Ni- and Cr- plated. Brass was chosen to ensure sufficient thermo conductivity. A space of $1 \mathrm{~mm}$ was needed between the trough chamber and the solvent reservoir to prevent disappearance of solvent by capillary action between these parts. Troughs in the VP-chamber (Scheme 1) were each filled with about $5 \mathrm{ml}$ of the appropriate liquid mixture, and then the plate was fixed in position. After a $10 \mathrm{~min}$ equilibrium period, the solvent reservoir $(40 \mathrm{~cm} \times 1 \mathrm{~cm} \times 2 \mathrm{~cm})$ was filled with $25 \mathrm{ml}$ of developing solvent. N-chambers contained $100 \mathrm{ml}$ of developing solvent and were saturated with solvent vapour by lining the walls with filter paper. After 45-60min the plate was introduced and development started. The developing solvent was allowed to run $16 \mathrm{~cm}$ beyond the starting point, whereas in VP-TLC continuous development was used for the time given in minutes. All experiments were carried out at $21 \pm 1{ }^{\circ} \mathrm{C}$ at a relative humidity of $27-41 \%$. Within this range the separations were reproducible.

\section{Detection and photography}

Detection was carried out in UV light of 254nm (Camag, Universal lamp), followed by photography under two such lamps on Agfacolor CT 18 diapositive film with an Asahi Pentax type SV camera with $49 \mathrm{~mm}$ UV ghostless filter. Exposure was $3 \mathrm{sec}$, distance $70 \mathrm{~cm}$ and aperture 5.6. 


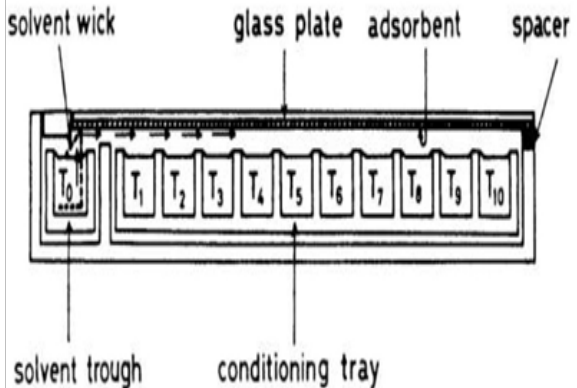

Scheme I Cross-sectional view of the chamber for vapour-programming (VP-TLC).

\section{Results}

With the conventional TLC technique using ether-methanol mixtures as developing solvents, results are far from optimal. With ether-methanol (90:10) the first seven substances are reasonably separated but the remaining eight all show about the same migration. Furthermore, it should be noticed that only about $3 / 5$ of the plate is utilised for the spread. With the more polar developing solvent, ether-methanol (80:20), the substances move to higher positions on the plate and these gave a decreased resolution. The separations are illustrated in Figure 1. Furthermore, unsaturated chambers did not improve the separation significantly, probably due to the strong polarity of the methanol (displacing action). With the VP-chamber, however, a strikingly improved result could be obtained with ethermethanol (95:5) as developing solvent and ether-methanol mixtures in the accelerating troughs. Benzene-ether (50:50) had to be used as a retarding liquid in order to suppress tailing of the spots. The separation and the vapour programme are shown in Figure 1.
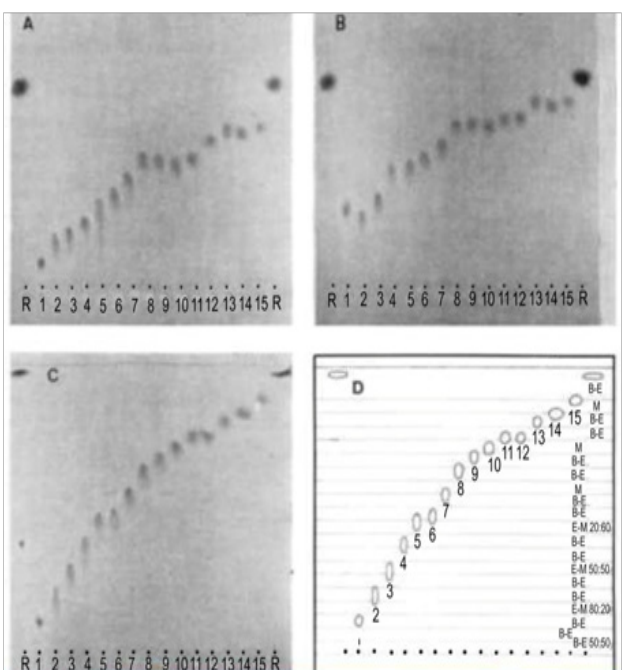

Figure I Improved separation of sulphonamides in the vapour-programming chamber as compared to separations in saturated $\mathrm{N}$-chambers. A) $\mathrm{N}$-chamber, developing solvent ether-methanol $(90: 10)$. Temperature $21.5^{\circ} \mathrm{C}$, relative humidity $27 \%$, saturation $45 \mathrm{~min}$, development $40 \mathrm{~min}$. B) N-chamber, developing solvent ether-methanol $(80: 20)$, temperature $21.5^{\circ} \mathrm{C}$, relative humidity $27 \%$, saturation $45 \mathrm{~min}$, development $40 \mathrm{~min}$. C) VP-chamber, developing solvent ether-methanol (95:5), temperature $21.8^{\circ} \mathrm{C}$, relative humidity $30 \%$, saturation $10 \mathrm{~min}$, development $79 \mathrm{~min}$, spacers $0.5 \mathrm{~mm}$, cooling $19^{\circ} \mathrm{C}$. D) Schematic diagram of the positions of the troughs and the liquid compositions therein during development. B-E) benzene-ether (50: 50); E: Ether; M: Methanol; I: Sulphaguanidine; 2: Sulphamethizole; 3: Sulphisomidine; 4: Sulphathiazole; 5: Sulphadiazino; 6: Sulphacetamide; 7: Sulphamerazine; 8: Sulphadimidine; 9: Sulphapyridine; 10:Sulphamethoxypyrimidine; I I:Sulphamethoxypyridazine; I2: Sulphisoxazole; I 3:Sulphadimethoxine; | 4:Sulphanilamide; I 5: Sulphaphenazole; R: Reference 4-Nitroaniline;Adsorbent: Silica Gel GF ${ }_{254}$, Load $3 \mu$ g.
Only sulphamethoxypyridazine and sulphisoxazole do not separate under these conditions, but the other components are well separated and spread over the whole of the plate. Because of the amphoteric character of the substances under investigation which are, in fact, all sulphanilamides, we assumed that a separation in alkaline medium would be valuable but experiments with the classical N-chambers were very unsuccessful. The most suitable systems were chloroformmethanol-ammonia mixtures but as can be seen in Figure 2 results are rather poor. With the VP-chamber, a greatly improved resolution was again found on using the bottom layer of the chloroformmethanol-25\% ammonia mixture (70:20:10) as developing solvent and a chloroform-methanol-ammonia vapour programme.
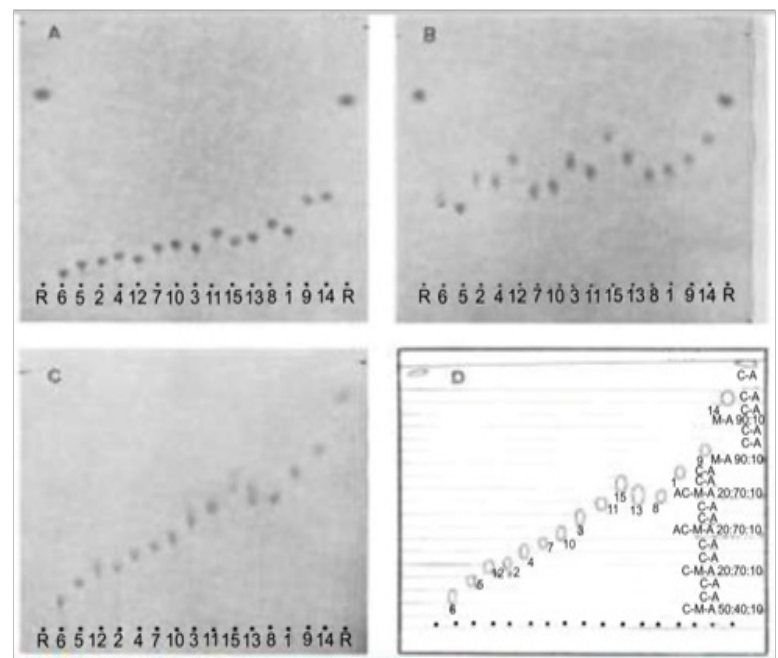

Figure 2 Improved separation of sulphonamides in the vapour-programming chamber as compared to separation in saturated $\mathrm{N}$-chambers. A) $\mathrm{N}$-chamber, developing solvent bottom layer of chloroform-methanol-25\% ammonia (70:20:10). Temperature $22^{\circ} \mathrm{C}$, relative humidity $41 \%$, saturation $60 \mathrm{~min}$, development $6 \mathrm{I}$ min. B) $\mathrm{N}$-chamber, developing solvent chloroformmethanol-25\% ammonia $(50: 40: 10)$, temperature $22.0^{\circ} \mathrm{C}$, relative humidity $41 \%$, saturation $10 \mathrm{~min}$, development $121 \mathrm{~min}$, spacers $1 \mathrm{~mm}$, cooling $19^{\circ} \mathrm{C}$. C) VP-chamber, developing solvent bottom layer of chloroform-methanol-25\% ammonia $(70: 20: 10)$, temperature $22.0^{\circ} \mathrm{C}$, relative humidity $41 \%$, saturation $10 \mathrm{~min}$, development $121 \mathrm{~min}$, spacers $1 \mathrm{~mm}$; cooling $19^{\circ} \mathrm{C}$. D) Schematic diagrams of the positions of the troughs and the liquid compositions herein during development. C: Chloroform; M: Methanol; A: 25\% Ammonia; AC: Acetone; C-A: Chloroform Saturated with 25\% Ammonia; I: Sulphaguanidine; 2: Sulphamethizole; 3: Sulphisomidine; 4: Sulphathiazole; 5: Sulphadiazino; 6 : Sulphacetamide; 7: Sulphamerazine; 8: Sulphadimidine; 9: Sulphapyridine; 10: Sulphamethoxypyrimidine; I I: Sulphamethoxypyridazine; I2: Sulphisoxazole; 13: Sulphadimethoxine; I4: Sulphanilamide; I5: Sulphaphenazole; R: Reference 4-Nitroaniline;Adsorbent: Silica Gel GF ${ }_{254}$, Load $3 \mu \mathrm{g}$.

Chloroform saturated with ammonia was a suitable decelerator. As can be seen in Figure 2 the spread is nearly $70 \%$ and only sulphadimethoxine and sulphadimidine coincide. It should be noted that the VP-systems described are suitable for two-dimensional development. Thus, it can be concluded that VP-TLC is highly effective in the analysis of sulphonamides. With the -two systems described identification of all 15 compounds becomes possible, which is by no means obtained with the conventional technique. The actual advantage of VP-TLC is that close-lying spots can be pulled apart and then guided to a position in the chromatogram which is not yet occupied by another compound. Accordingly, the entire plate length can be utilised to cover the spread of the spots. Furthermore, due to the fact that any desired vapour composition can be selected and applied to any point of the plate, the analyst can select the optimal conditions for a particularly difficult separation of two compounds, without disturbing the separation already obtained of the other components 
present in the sample. This will be particularly valuable in separations of closely related substances.

\section{Discussion}

We indeed postulated that the increase in resolution which can be obtained in unsaturated chambers could possibly be due to a concentration gradient of vapour in the dry adsorbent. In order to be able to control such a gradient and/or to improve the gradient, we thus developed the vapour-programming chamber. We made such vapour programmes that a concentration gradient of adsorbed vapour on the dry plate should be obtained and this system did work, i.e. we could distinctly improve greatly many separations. This can be more practical if the composition of the liquid present on the plate at the end of development is known without any need to indicate the composition of the adsorbed vapour on the dry plate. We postulated that the adsorbed vapour would be very important as a precursor of the stationary phase, but this stationary phase cannot be determined in the way Geiss described. ${ }^{3}$ His determination is that of the mobile and the stationary phases so that no conclusion can be drawn with regard to the latter.

Furthermore the Vario-KS-chamber cannot be compared with the VP-chamber. The two chambers have been developed for two different purposes, each having its own requirements. If VP-TLC is not carried out properly, e.g. by using a different chamber, negative results may well occurs.

Jänchen, ${ }^{4}$ as Kadkhodayan \& Brenner $^{5}$ pointed out, van Hout et al. ${ }^{6}$ can deliberately choose the preloading liquids and can arrange them, if they wish, in an alternating series. However, such very, empirical arrangements make it extremely difficult to understand the separation mechanism. One can even overlook important parameters which in reality govern the chromatographic behaviour. We believe Geiss $^{3}$ would give us more details illustrating what we mean. van Hout et al. ${ }^{6}$ had just developed VP-TLC because of the increasing demand for better separation techniques. It must be said, however, that this empirical system shows satisfactory reproducibility. Of course we would highly appreciate it if somebody could give us a theoretical explanation for the system, but in our opinions it would not be very advisable to work out a theoretical explanation first before using a separation technique. Even normal TLC procedures are still not fully understood at the moment but we think it cannot be denied that TLC has been highly appreciated during the past decade. Jänchen ${ }^{4}$ thought that good separations are in fact desirable. What is even more important, however, are reliable or consistent separations which are much easier to achieve with preloading gradients, perhaps superimposed gradients, rather than with alternating preloading steps. van Hout et al. ${ }^{6}$ showed that it satisfactory when separation can be obtained by simpler techniques than VP-TLC, of course we would by no means advocate the use of a more complicated system. However, VP-TLC has been especially devised to be used where no result can be obtained with other TLC techniques. van Hout et al. ${ }^{6}$ has stated that the Vario-KS-chamber cannot be used for VP-technique, one of the reasons being too large a distance between layer and conditioning trough walls and another one being the fact that the Vario-KS-chamber has only ten subdivisions. We should like to ask (a) what the ideal distance would be, and (b) why it is important for the VP-technique. In general, those more than ten subdivisions are used.

According to van Hout et al., ${ }^{6}$ (a) It is impossible to indicate just one optimum distance between the plate and the trough walls. This changes from system to system and is dependent on its polarity. With more polar systems the distance should be larger due to greater swelling of the adsorbent; with less polar systems the distances should be decreased. (b) The presence of only ten troughs would prevent an optimum vapour programme because you are too limited in choosing and altering vapour conditions.

Jänchen ${ }^{4}$ sees that (a) The distance between layer and upper part of the conditioning trays in the Vario-KS-chamber is $1.0 \mathrm{~mm}$ minus layer thickness and can be reduced to $0.5 \mathrm{~mm}$ minus layer thickness by inserting the "manipulating sheet" under the tray. This, in our opinion, is more than sufficient, particularly when one bears in mind that in most cases with the Vario-KS-technique a separation sheet is inserted between layer and trays during development. This is important because it prevents the mobile phase from interacting with the conditioning liquids in an unpredictable and uncontrollable manner. (b) It is agreed that for applying alternating preloading liquids more than 10 troughs may be desirable. However, for applying a certain influence decreasing or increasing in one direction 10 troughs provide a sufficient number of subdivisions to establish a quasi-continuous gradient.

According to Ramić et al., ${ }^{7}$ when one component of the developing solvent mixture comes from the vapour phase onto the plate, which is the case in this work, it changes the characteristics of the mobile phase; it causes the change of distribution coefficient $\alpha=\sigma_{\mathrm{S}} / \sigma_{\mathrm{M}}$. The concentration of the developing solvent sorbed from the vapour phase into the mobile phase is proportional to the partial pressure, and because the RF value is a function of $\alpha$, we must control the partial pressure of the solvent. If this control is not possible, $\mathrm{RF}$ values are not reproducible.

Kadkhodayan \& Brenner ${ }^{5}$ accepted the view of Ramić et al. ${ }^{7}$ that could conclude that a chamber with a maximum of compartments perpendicular to the direction of developing solvent flow and a minimum possible distance (just omitting capillary effects) between the top of the septa and the moist layer of the sorbent should give best results. Of course, this conclusion is not valid in the case that preloading the layer from the vapour phase has additional effects besides its influence on the characteristics of the mobile phase. Dynamic factors must not be neglected. It is, however, difficult to assess them. We recommend therefore that van Hout et al. ${ }^{6}$ approach be regarded, just as the humidity approach of Geiss, as an empirical one. In addition to the remarks of Kadkhodayan \& Brenner ${ }^{5}$ we would like to say that it is one of the main principles of VP-TLC that the mobile and stationary phases are changed during development. Of course this makes theoretical treatment more difficult, but it has been shown that the practical advantages of influencing the phases during development are quite distinct.

Kadkhodayan \& Brenner ${ }^{5}$ has criticised of van Hout et al. ${ }^{6}$ method for theoretical reasons is beyond the point because the method does not claim to have a theoretical background. The programme for preloading the layer with developing solvent vapours is derived in a purely empirical manner. It is experimentally built up in such a way as to secure the desired spreading of the spots over the entire length of the chromatogram. There may be mixtures for which no satisfactory programme can be found within a reasonable amount of time. But once a programme is devised we really do not see why we should doubt reproducibility of separation results. The entire problem is essentially no more complicated than the search for a developing solvent and its use in chemical TLC. 
Now let us say a word on the limits of application of theory. They are given by the fact that chromatographic separation depends on two factors and both of them must be known for theoretical consideration. These are the partition equilibrium and the absolute rate of movement of the developing solvent. The importance of the latter was discussed on occasion of the third Symposium in Liblice. Let us just remind you that in any given case it determines the relative rate of solute transport and diffusion, and let us further remind you that TLC separation effects are most dramatic at the very beginning of a run when developing solvent migration rate is at a maximum. This fact is, by the way, the basis of high pressure chromatography mentioned by Wen et al. ${ }^{1}$

In van Hout et al. ${ }^{6}$ systems we know neither how the preloading of the layer from the vapour phase influences the rate and the local uptake of liquid mobile phase by a given section of the layer nor how it affects developing solvent flux between starting point and solvent fronts. In other words, there are no data available on the local nature of the chromatographic system (composition, phase ratio) or on the local rate of solvent flow. So let us forget theory in this case. Instead let us be aware that programmed preloading represents an ingenious manner to enhance the separation power of TLC.

But, how does such a vapour-programmed TLC compare e.g. with a suitable multiple developing technique? Multiple developments were not suitable in this case and, in general, could not be used successfully in other separations as well. It may be that for two or three components multiple developments give satisfactory separations, but for the group as a whole the multiple developments will not be suitable. The vapour-programme, on the other band, can be completely adapted to the various separation problems.

Melzacka \& Shellard ${ }^{8}$ concerned the separation of sulphonamides. They gave no evidence of actual separation of mixtures. Did they attempt to separate a mixture of, say, three sulphonamides of fairly close RF values? van Hout et al. ${ }^{6}$ achieved excellent separations of mixtures that obtained with the described procedure. The reproducibility of the method is good and the migration rates together with the aid of known reference substances provide suitable identification possibilities. This also holds for closely lying substances.

\section{Acknowledgements}

None.

\section{Conflict of interest}

The author declares no conflict of interest.

\section{References}

1. Wen E, Lu M, Zhou X, et al. High-performance thin-layer chromatographic quantification of three active compounds in Euphorbia humifusa Willd. and TLC-DPPH test for screening antioxidant components. $J$ Planar Chromatogr Mod TLC. 2016;29(6):417-422.

2. Sjursnes BJ, Kvittingen L, Schmid R. Thin-layer chromatography of green leaves extracts: Zinc migrates into pheophytin on TLC silica plates with fluorescence indicator $\left(\mathrm{F}_{254}\right)$. J Planar Chromatogr Mod TLC. 2016;29(6):480-483.

3. Perry JA, Jupille TH, Glunz LJ. TLC [thin-layer chromatography]. Programmed multiple development. Anal Chem. 1975;47(1):65A-74A

4. Jänchen J, van Wolput JHMC, van Well WJM, et al. Adsorption of water, methanol and acetonitrile in $\mathrm{ZK}-5$ investigated by temperature programmed desorption, microcalorimetry and FTIR. Thermochim Act. 2001;379(1-2):213-225.

5. Kadkhodayan A, Brenner A. ChemInform abstract: Temperature-Programmed Reduction and Oxidation (TPR/TPO) of metals supported on $\gamma$-Alumina. ChemInform. 1989:20(39)

6. van Hout MWJ, van Egmond WMA, Franke JP, et al. Feasibility of the direct coupling of solid-phase extraction-pipette tips with a programmed-temperature vaporiser for gas chromatographic analysis of drugs in plasma. J Chromatogr B Analyt Technol Biomed Life Sci B. 2002; 766(1):37-45.

7. Ramić A, Medić-Šarić M, Turina S, et al. TLC detection of chemical interactions of vitamins A and D with drugs. J Planar Chromatogr Mod TLC. 2006;19(107):27-31.

8. Melzacka M, Shellard EJ. Separation of the degradation products of 1-benzoyl-5, 5-diethylbarbituric acid by means of vapour programmed thin-layer chromatography. J Chromatogr A. 1970;49:541543 